\title{
Azimuthal magnetic fields in Saturn's magnetosphere: effects associated with plasma sub-corotation and the magnetopause-tail current system
}

\author{
E. J. Bunce, S. W. H. Cowley, and J. A. Wild \\ Department of Physics and Astronomy, University of Leicester, Leicester LE1 7RH, UK
}

Received: 5 November 2002 - Revised: 6 March 2003 - Accepted: 18 March 2003

\begin{abstract}
We calculate the azimuthal magnetic fields expected to be present in Saturn's magnetosphere associated with two physical effects, and compare them with the fields observed during the flybys of the two Voyager spacecraft. The first effect is associated with the magnetosphereionosphere coupling currents which result from the subcorotation of the magnetospheric plasma. This is calculated from empirical models of the plasma flow and magnetic field based on Voyager data, with the effective Pedersen conductivity of Saturn's ionosphere being treated as an essentially free parameter. This mechanism results in a 'lagging' field configuration at all local times. The second effect is due to the day-night asymmetric confinement of the magnetosphere by the solar wind (i.e. the magnetopause and tail current system), which we have estimated empirically by scaling a model of the Earth's magnetosphere to Saturn. This effect produces 'leading' fields in the dusk magnetosphere, and 'lagging' fields at dawn. Our results show that the azimuthal fields observed in the inner regions can be reasonably well accounted for by plasma sub-corotation, given a value of the effective ionospheric Pedersen conductivity of $\sim 1-2$ mho. This statement applies to field lines mapping to the equator within $\sim 8 R_{S}\left(1 R_{S}\right.$ is taken to be $\left.60330 \mathrm{~km}\right)$ of the planet on the dayside inbound passes, where the plasma distribution is dominated by a thin equatorial heavy-ion plasma sheet, and to field lines mapping to the equator within $\sim 15 R_{S}$ on the dawn side outbound passes. The contributions of the magnetopause-tail currents are estimated to be much smaller than the observed fields in these regions. If, however, we assume that the azimuthal fields observed in these regions are not due to sub-corotation but to some other process, then the above effective conductivities define an upper limit, such that values above $\sim 2$ mho can definitely be ruled out. Outside of this inner region the spacecraft observed both 'lagging' and 'leading' fields in the post-noon dayside magnetosphere during the inbound passes, with 'leading' fields being observed both adjacent to the magnetopause and in the ring current re-
\end{abstract}

Correspondence to: E. J. Bunce (emma.bunce@ion.le.ac.uk) gion, and 'lagging' fields being observed between. The observed 'lagging' fields are consistent in magnitude with the sub-corotation effect with an effective ionospheric conductivity of $\sim 1-2$ mho, while the 'leading' fields are considerably larger than those estimated for the magnetopause-tail currents, and appear to be indicative of the presence of another dynamical process. No 'leading' fields were observed outside the inner region on the dawn side outbound passes, with the azimuthal fields first falling below those expected for sub-corotation, before increasing, to exceed these values at radial distances beyond $\sim 15-20 R_{S}$, where the effect of the magnetopause-tail currents becomes significant. As a by-product, our investigation also indicates that modification and scaling of terrestrial magnetic field models may represent a useful approach to modelling the three-dimensional magnetic field at Saturn.

Key words. Magnetospheric physics (current systems; magnetosphere-ionosphere interactions; solar windmagnetosphere interactions)

\section{Introduction}

Analysis of magnetometer data obtained during the three flybys of Saturn's magnetosphere undertaken to date, by Pioneer-11 in 1979, and by Voyagers-1 and -2 in 1980 and 1981, respectively, have revealed a number of surprising features of Saturn's magnetic field. The main surprise has been that, within the limitations of the data coverage, the internal field of the planet has been found to be closely symmetric about the spin axis, consisting of the sum of axisymmetric dipole, quadrupole, and octupole terms (Connerney et al., 1982, 1984; Davis and Smith, 1990). The internal field is, therefore, entirely poloidal, with no significant azimuthal components relative to the spin axis. Nevertheless, small-amplitude azimuthal fields were observed inside the magnetosphere, $\sim 5-10 \mathrm{nT}$ in magnitude, which have been attributed to a number of effects. One such feature, de- 

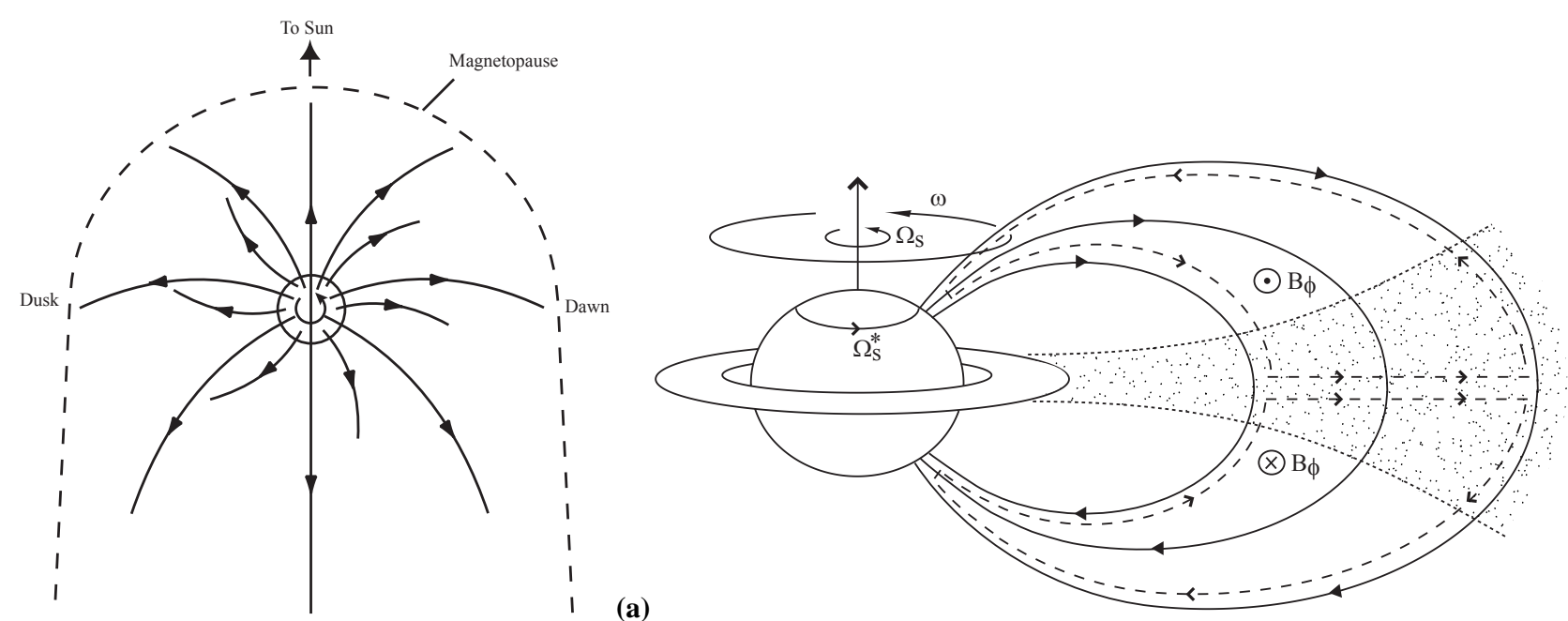

(b)

Fig. 1. (a) Sketch looking down onto the northern pole of Saturn, showing field lines (arrowed solid lines) projected onto the equatorial plane. The sense of planetary rotation is anti-clockwise, as indicated. The inner field lines are shown bent into a 'lagging' configuration associated with angular momentum transfer from the atmosphere to the magnetosphere due to sub-corotation of the magnetospheric plasma. The outer field lines are shown bent away from noon, due to the effect of the magnetopause (solid line) and tail current system. (b) Sketch of a meridian cross section through Saturn's quasi-axisymmetric inner and central magnetosphere, extending to distances of $\sim 15-20 R_{S}$ in the equatorial plane. The arrowed solid lines indicate magnetic field lines, which are modestly distended outward from the planet by azimuthal currents (the 'ring-current') flowing in the near-equatorial plasma. The rotating plasma is shown by the dotted region, consisting mainly of protons and oxygen ions and associated electrons, which derive from water ice originating from ring grains and moon surfaces. Three separate angular velocities are indicated. These are the angular velocity of a particular shell of field lines $\omega$, the angular velocity of the planet $\Omega_{S}$, and the angular velocity of the neutral upper atmosphere in the Pedersen layer of the ionosphere, $\Omega_{S}^{*}$. The value of $\Omega_{S}^{*}$ is expected to lie between $\omega$ and $\Omega_{S}$ because of the frictional torque on the atmosphere due to ion-neutral collisions. The oppositely-directed frictional torque on the magnetospheric flux tubes is communicated to the equatorial plasma by the current system indicated by the arrowed dashed lines, shown here for the case of sub-corotation of the plasma (i.e. $\omega<\Omega_{S}$ ). This current system bends the field lines out of meridian planes into a 'lagging' configuration, as shown by the inner field lines in (a), associated with the azimuthal field components $B_{\varphi}$, as shown.

scribed by Connerney et al. (1983), was observed during the Voyager-1 flyby. In this case, positive azimuthal fields peaking at $\sim 10 \mathrm{nT}$ were observed near the closest approach, varying inversely with the distance from the planet's spin axis, in apparent association with additional perturbations in the radial and latitudinal field components. Connerney et al. (1983) modelled these field perturbations using a fieldaligned line current of $\sim 10^{7} \mathrm{~A}$, which flows along a dipole field line into the southern nightside ionosphere of the planet at $80^{\circ}$ southern latitude and 21:30 LT (Local Time). Current closure was taken to be via an ionospheric auroral electrojet followed by outflow in the early morning sector, though the data were found not to be sensitive to the exact closure path. It was also noted by Connerney et al. (1983) that this represented one simple solution for the current system responsible, and that other configurations were possible. The physical origin of the current system was taken to be related to solar wind-induced magnetospheric convection. Other features in the azimuthal field were attributed by Connerney et al. (1983) to the effect of field line bending due to the magnetopausetail current system. In addition to these effects, Espinosa and Dougherty (2000) have more recently pointed out the intermittent existence of oscillations in the radial and azimuthal components of the field, which are of $\sim 5 \mathrm{nT}$ amplitude and vary on the planetary spin period of $\sim 11 \mathrm{~h}$. Such features were clearly observed during the Pioneer-11 flyby, inbound and outbound, and may also have been present during the Voyager-2 inbound pass, but were absent during the Voyager2 outbound pass and throughout the Voyager- 1 flyby. With the above internal field description, however, whatever their origin, they clearly cannot have been due directly to variations of the internal field modulated by the spin of the planet.

Another source of azimuthal fields in Saturn's magnetosphere which has not been quantitatively investigated to date, though briefly discussed by Connerney et al. (1983), arises from the effect of plasma mass-loading and radial transport in the corotation-dominated inner and central regions of the magnetosphere. Such azimuthal fields are very evident, for example, in Jupiter's middle magnetosphere (e.g. Smith et al., 1976; Acuña et al., 1983; Bunce and Cowley, 2001; Khurana, 2001), associated with angular momentum transfer to plasma flowing outward from the Io torus (e.g. Hill, 1979, 2001; Vasyliunas, 1983; Khurana and Kivelson, 1983; Cowley and Bunce, 2001). In the case of Saturn, water-group molecules which originate from ring grains and moon surfaces are ionised and picked up by the corotating flow, and are subsequently lost mainly either by charge-exchange or radial transport out of the system (e.g. Richardson, 1992; 
Richardson et al., 1998). These processes cause the magnetospheric plasma to rotate at angular velocities less than that of the planet, which, in turn, causes the field lines to bend into a 'lagging' configuration associated with angular momentum transfer from the atmosphere to the magnetospheric plasma. This sense of field bending is illustrated by the inner field lines in Fig. 1a, which shows a view looking down onto the north pole of the planet. For the case of Saturn, where the field is directed southward at the equator, the sense of the associated azimuthal field is negative north of the equator, reversing to positive south of the equator. The associated system of electric currents is illustrated by the dashed lines in Fig. 1b. Pedersen currents flow equatorward in the ionosphere, driven by the equatorward-directed electric field of the sub-corotating flow in the neutral atmosphere rest frame, and close via field-aligned currents in equatorial currents which flow radially outward in the magnetospheric plasma. The magnetic signature of this current system is then the azimuthal components marked $B_{\varphi}$ in the figure, which are associated with the 'lagging' field configuration shown in the inner field lines in Fig. 1a. In mechanical terms, the torque associated with the $\boldsymbol{j} \times \boldsymbol{B}$ force of the Pedersen current balances the torque on the ionosphere due to ion-neutral collisions in the Pedersen layer, while the equal and opposite torque associated with the $\boldsymbol{j} \times \boldsymbol{B}$ force of the equatorial current accelerates the magnetospheric plasma in the sense of corotation. Overall, angular momentum is transferred from the planet's atmosphere to the magnetospheric plasma. Observations from the Voyager spacecraft have demonstrated that the plasma in the central and outer regions of Saturn's magnetosphere does indeed significantly sub-corotate with respect to the planet (Richardson, 1986; Richardson and Sittler, 1990). Consequently, corotation-enforcement currents of the above nature must flow in Saturn's magnetosphere, such that the azimuthal fields they produce must contribute at some level to those observed.

A second effect which must also contribute to the observed azimuthal field at some level, but which has also not been investigated quantitatively, is the effect of the day-night asymmetric confinement of the planetary field by the solar wind, i.e. the effect of the magnetopause and tail current system. The sense of the field bending associated with these currents is shown by the outer field lines in Fig. 1, where the field lines are bent away from noon on either side, such as has long been established to take place in the Earth's magnetosphere (Fairfield, 1968). The sense of the bending is the same as that of the 'lagging' fields due to plasma sub-corotation in the dawn sector, but is in the opposite sense in the dusk sector, such that the effect produces a 'leading' field configuration in this case. It should be emphasised, however, that in the dayside and quasi-dipolar regime, the overall field perturbations due to this effect are curl-free and not related to local stresses on the plasma, representing the 'fringing' fields of the magnetopause and tail currents.

The principal purpose of this paper is to make quantitative estimates of the azimuthal fields inside Saturn's magnetosphere due to the two effects discussed above, and to compare them with the fields observed during the spacecraft flybys. The calculation of the azimuthal field due to sub-corotation of the plasma is based on an analysis of the magnetosphere-ionosphere coupling current system outlined above, using theory presented in the companion paper by Cowley and Bunce (2003). Relevant results are briefly reviewed in the next section. The calculation requires knowledge of the angular velocity profile of the magnetospheric plasma. This information is available for the Voyager flybys, obtained from analysis of data from the PLS instruments (Richardson, 1986; Richardson and Sittler, 1990). However, only rudimentary angular velocity information is available for the Pioneer-11 flyby (Frank et al., 1980), such that we are unable to undertake a similar analysis of this data. Here, therefore, we concentrate on the data obtained from the Voyager flybys. We also require knowledge of the magnetic field, and for simplicity we must use an axisymmetric model. Therefore, we employ the SPV internal field model of Davis and Smith (1990) and the ring current model of Connerney et al. (1983). However, in order to model the solar wind-related effects, we explicitly require a non-axisymmetric model and thus, we simply scale a data-based model of the terrestrial magnetic field to Saturn. The validity of this procedure follows from the fact that the effects are essentially due to the confinement of the planetary field inside an asymmetric cavity, so that the large-scale geometry of the field lines due to this effect should be broadly the same in the two cases. In the following sections we outline the basis on which the azimuthal fields due to the above two effects have been calculated, and then present a comparison with the fields observed.

\section{Azimuthal field due to plasma sub-corotation}

In this section we calculate the azimuthal field associated with angular momentum transfer to sub-corotating magnetospheric plasma. We consider the value of the field at a point on a field line lying between the ionosphere and the closure currents in the equatorial region. If we apply Ampère's law to a circular path passing through the point, centred on Saturn's spin (and magnetic) axis, we find immediately

$B_{\varphi}=\mp \frac{\mu_{o} I_{h P}}{2 \pi \rho}$,

where the upper and lower signs correspond to conditions north and south of the equatorial current region. In this expression $I_{h P}$ is the equatorward-directed azimuth-integrated ionospheric horizontal Pedersen current flowing at the feet of the field lines in the hemisphere corresponding to the point in question, and $\rho$ is the perpendicular distance from the point to the spin axis (i.e. the cylindrical radial distance). The azimuthal magnetic field in the magnetosphere thus varies on a given field line inversely with the perpendicular distance from the spin (magnetic) axis, outside of the region where the equatorial closure currents flow. Within the region of the closure currents, of course, the magnitude of the azimuthal field will be reduced below that given by Eq. (1), toward zero 
near the equatorial plane where the azimuthal field reverses in sign. In deriving Eq. (1) we are, of course, assuming axial symmetry of the system about the spin axis. This should be a reasonable approximation to radial distances of $\sim 15$ $20 R_{S}$ in the equatorial plane, depending on the degree of extension of the magnetosphere, compared, for example, with distances of $\sim 17-24 R_{S}$ to the subsolar magnetopause (Behannon et al., 1983; Maurice and Engel, 1995). Here, $R_{S}$ is Saturn's radius which we take to be $60330 \mathrm{~km}$. This value for Saturn's radius is approximately the radial distance of the 1 bar level at the equator, and has been used widely in previous literature. For simplicity we use the same value here. However, we note that the present value of the radial distance of the 1 bar level at the equator recommended by the IAU is actually $60268 \mathrm{~km}$.

The azimuth-integrated horizontal Pedersen current $I_{h P}$ appearing in Eq. (1) is derived in the companion paper by Cowley and Bunce (2003), following earlier related calculations in the jovian context by Hill (1979) and Vasyliunas (1983). It is given by

$I_{h P}(\theta)=2 \pi \Sigma_{P}^{*} \Omega_{S} B_{i p} R_{i}^{2}(\theta) \sin ^{2} \theta\left(1-\frac{\omega}{\Omega_{S}}\right) f_{B}(\theta)$,

where $\Sigma_{P}^{*}$ is the effective height-integrated Pedersen conductivity of Saturn's ionosphere, $\Omega_{S}$ is Saturn's angular velocity $\left(1.638 \times 10^{4} \mathrm{rad} \mathrm{s}^{-1}\right), B_{i p}$ is the ionospheric field strength at Saturn's pole due to the dipole term alone ( $\sim 54760 \mathrm{nT})$, $R_{i}(\theta)$ is the radial distance of the conducting layer of the ionosphere as a function of co-latitude $\theta$ from the north pole, $\omega$ is the plasma angular velocity on the field line, and $f_{B}(\theta)$ is a magnetic field factor of order unity which contains the effects on the ionospheric current of the non-dipole field terms, the non-vertical field, and the variation of the field with latitude (see Cowley and Bunce (2003) for further details). The effective conductivity $\Sigma_{P}^{*}$ is related to the true value $\Sigma_{p}$ by

$\Sigma_{P}^{*}=(1-k) \Sigma_{p}$,

where parameter $k$ relates to the slippage of the angular velocity of the neutral atmosphere in the Pedersen layer $\Omega_{S}^{*}$, relative to the planet $\Omega_{S}$, due to the frictional torque associated with ion-neutral collisions, i.e.

$\left(\Omega_{S}-\Omega_{S}^{*}\right)=k\left(\Omega_{S}-\omega\right)$,

for some $0<k<1$ (see Fig. 1b and, e.g. Huang and Hill (1989) for a discussion in the jovian context).

In order to evaluate $I_{h P}$ from Eq. (2), and hence, to determine the azimuthal field from Eq. (1), models of four system parameters need to be established. The first is the radial distance of the conducting layer of the ionosphere from the planet's centre, $R_{i}(\theta)$. This is taken to be an ellipse

$$
R_{i}(\theta)=\frac{R_{e}}{\left(1+\varepsilon \cos ^{2} \theta\right)^{1 / 2}} \text { where } \varepsilon=\left(\frac{R_{e}}{R_{p}}\right)-1,
$$

such that the radial distance varies from $R_{p}=55364 \mathrm{~km} \approx$ $0.92 R_{S}$ at the poles to $R_{p}=61268 \mathrm{~km} \approx 1.02 R_{S}$ at the equator. These distances correspond to $1000 \mathrm{~km}$ above the 1 bar reference spheroid (see the discussion in the companion paper).

The second is a model of the magnetic field, both required to define field quantities in the ionosphere, and to map field lines between the magnetosphere and the ionosphere. Here, the ionospheric field has been taken to be given by the SPV internal field model of Davis and Smith (1990), consisting of the sum of axisymmetric dipole, quadrupole and octupole terms, as indicated in the Introduction. In the magnetosphere, the field of an axisymmetric ring current has also been added to the internal field, as derived by Connerney et al. (1983) from fits to the Voyager magnetometer data. Details are given in Sect. 2 of the companion paper.

The third requirement is a model of the angular velocity of the plasma normalised to the planetary angular velocity, $\left(\omega / \Omega_{S}\right)$. Here, we will use models derived from an analysis of data from the PLS instruments on the Voyager spacecraft, as detailed in the companion paper in Sect. 3. The data were obtained from the inbound pass of Voyager1 spanning the radial distance range $\sim 5-17 R_{S}$ at $\sim 13: 00$ 15:00 MLT, the outbound pass of Voyager-1 spanning $\sim 4$ $8 R_{S}$ at $\sim 02: 00 \mathrm{MLT}$, and the inbound pass of Voyager2 spanning $\sim 4-20 R_{S}$ at $\sim 13: 00-15: 00$ MLT (Richardson, 1986; Richardson and Sittler, 1990). However, the inbound and outbound Voyager-1 data have been combined together on account of the relative sparseness of the data in the inner region, and on the basis that axisymmetry should form a good approximation in this region. Overall, these data show that the angular velocity of the plasma declines from near-rigid corotation with the planet (i.e. $\left.\left(\omega / \Omega_{S}\right) \approx 1\right)$ in the inner region, to values of roughly half of rigid corotation at large distances (see Fig. 4a of the companion paper). However, as previously pointed out by Richardson (1986), the data suggest that the departure from rigid corotation starts closer to the planet for Voyager-1 than for Voyager-2. We have, therefore, constructed separate models for the two flybys. The models are parameterised in terms of the flux function of the magnetic field, $F$, related to the poloidal field components by $\boldsymbol{B}=(1 / \rho) \nabla F \times \hat{\boldsymbol{\varphi}}$ (in cylindrical co-ordinates referenced to the spin (and magnetic) axis of the planet). This function is such that $F=$ const defines a shell of field lines mapping between the northern and southern ionospheres via the equatorial plane. Definition of the angular velocity as a function of $F$ thus defines the plasma angular velocity at all points within the quasi-axisymmetric magnetosphere. The functional form employed to model the large-scale behaviour of the angular velocity is

$\left(\frac{\omega}{\Omega_{S}}\right)=\left(\frac{\omega}{\Omega_{S}}\right)_{\infty}+\left(1-\left(\frac{\omega}{\Omega_{S}}\right)_{\infty}\right) \frac{1}{\left(1+\left(F_{o} / F\right)^{n}\right)}$,

such that the plasma rigidly corotates at small radial distances, i.e. $\left(\omega / \Omega_{S}\right) \rightarrow 1$ as $F \rightarrow \infty$, while the angular velocity monotonically decreases to $\left(\omega / \Omega_{S}\right)_{\infty}$ at large distances where $F \rightarrow 0$. Parameter $F_{o}$ determines the radial location where the angular velocity change occurs, while parameter $n$ determines the gradient of the change, becoming sharper with increasing $n$. The parameter values cho- 


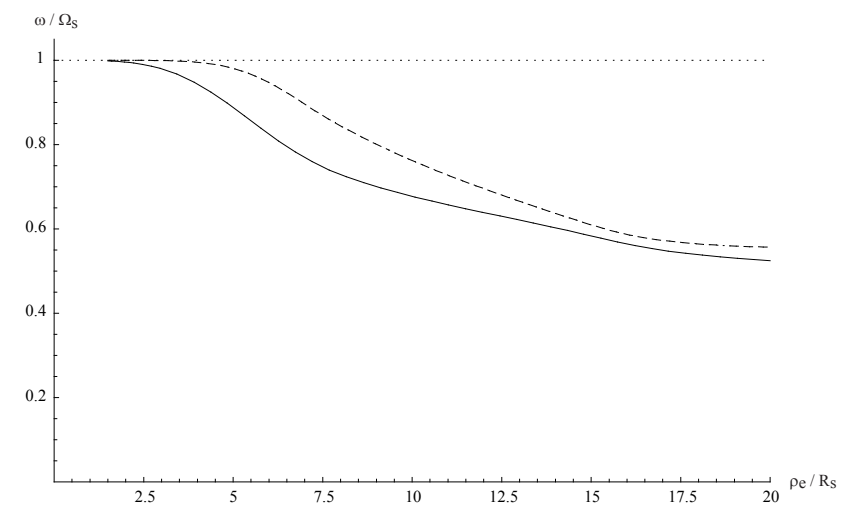

Fig. 2. Plot of the model $\left(\omega / \Omega_{S}\right)$ profiles mapped along field lines into the equatorial plane and shown as a function of radial distance $\rho_{e}$ from the planet, normalised to the value of Saturn's radius taken here to be equal to $60330 \mathrm{~km}$. The solid line is the large-scale model for Voyager-1 (with small-scale features considered in the companion paper by Cowley and Bunce (2003) omitted), while the dashed line is the corresponding model for Voyager-2. The dotted line shows the condition for rigid corotation, i.e. $\left(\omega / \Omega_{S}\right)=1$.

sen to represent the Voyager-1 data are $\left(\omega / \Omega_{S}\right)_{\infty}=0.5$, $F_{o}=3200 \mathrm{nT} R_{S}^{2}$ (corresponding to $7.4 R_{S}$ in the equatorial plane, see Fig. 2a of the companion paper), and $n=4$, while those for Voyager- 2 are $\left(\omega / \Omega_{S}\right)_{\infty}=0.55, F_{o}=2800 \mathrm{nT} R_{S}^{2}$ (corresponding to $9.7 R_{S}$ in the equatorial plane), and $n=7$. The presence of small-scale features in the Voyager-1 profile, discussed in the companion paper, represent an unnecessary refinement here and have not been included. In Fig. 2 we thus show the two model angular velocity profiles representing the large-scale behaviour, plotted versus distance in the equatorial plane. The profile for Voyager- 1 is shown by the solid line, while that for Voyager-2 is shown by the dashed line. It can be seen that the model Voyager-2 profile declines from near-rigid corotation at larger radial distances from the planet than the Voyager-1 profile, as indicated above.

The fourth parameter we require is the effective heightintegrated Pedersen conductivity of Saturn's ionosphere, possibly reduced from the true value by atmospheric 'slippage', as previously discussed. However, this is a very uncertain parameter at the present time. Estimates of the true value based on observed and modelled ionospheric electron density profiles have yielded values varying between $\sim 0.1$ and $\sim 100$ mho (Atreya et al., 1984; Connerney et al., 1983; Cheng and Waite, 1988). Here, therefore, we regard $\Sigma_{P}^{*}$ effectively as a free parameter, which we wish to estimate, or set limits on, from fits of Eq. (1) to observed $B_{\varphi}$ data. This comparison is carried out in Sect. 4.

\section{Estimating the azimuthal field due to magnetopause and tail currents}

Here, we now outline the method we have employed to estimate the azimuthal magnetic field in Saturn's magnetosphere, which results from the effects of the magnetopause and tail currents. Our procedure is essentially empirical, based on scaling a data-based model of the Earth's magnetospheric magnetic field to Saturn. The theoretical basis of the calculation is that the effects of interest are essentially due to the confinement of the planetary field inside an asymmetric cavity formed by normal stress balance with the dynamic pressure of the solar wind, such that to a first approximation the geometry of the field lines should be preserved, relative to the magnetopause, in the two systems.

Specifically, we have used the Tsyganenko $(1995,1996)$ field model, in which we have set the Earth's dipole to be anti-aligned with the spin axis, and have scaled the axial quadrupole and octupole fields to mimic Saturn. All other harmonic coefficients of the internal field have been set to zero. We have also chosen a 'standard' Earth field model, with IMF $B_{z}$ equal to zero, a solar wind dynamic pressure of $2 \mathrm{nPa}$ (essentially the average value at Earth), and we consider equinox conditions with the spin and dipole axis perpendicular to the planet-Sun line (Saturn was near to equinox during the Voyager flybys). To scale this Earth field to Saturn we then simply scale positions in relation to the observed radius of the sub-solar magnetopause, and the field magnitude and direction such that at small radial distances the scaled field simply becomes the SPV internal field model. That is to say we assume

$$
\begin{aligned}
& \boldsymbol{r}_{E}=\left(\frac{R_{M E}}{R_{M S}}\right) \boldsymbol{r}_{S} \text { and } \\
& \boldsymbol{B}_{S}\left(\boldsymbol{r}_{S}\right)=\frac{g_{1 S}^{0}}{g_{1 E}^{0}}\left(\frac{R_{M E}}{R_{E}} \frac{R_{S}}{R_{M S}}\right)^{3} \boldsymbol{B}_{E}\left(\boldsymbol{r}_{E}\right),
\end{aligned}
$$

together with

$$
\begin{aligned}
& g_{2 E}^{0}=\left(\frac{g_{1 E}^{0}}{g_{1 S}^{0}}\right)\left(\frac{R_{M E}}{R_{E}} \frac{R_{S}}{R_{M S}}\right) g_{2 S}^{0} \text { and } \\
& g_{3 E}^{0}=\left(\frac{g_{1 E}^{0}}{g_{1 S}^{0}}\right)\left(\frac{R_{M E}}{R_{E}} \frac{R_{S}}{R_{M S}}\right)^{2} g_{3 S}^{0},
\end{aligned}
$$

where $\boldsymbol{r}_{E}$ is the equivalent position vector in the Earth's magnetosphere for a given position vector $\boldsymbol{r}_{S}$ in Saturn's magnetosphere, and $\boldsymbol{B}_{S}\left(\boldsymbol{r}_{S}\right)$ is the expected field at $\boldsymbol{r}_{S}$ based on the Earth's field $\boldsymbol{B}_{E}\left(\boldsymbol{r}_{E}\right)$ at $\boldsymbol{r}_{E}$. Parameter $R_{M E}$ is the radial distance of the Earth's subsolar magnetopause $\left(\sim 11.1 R_{E}\right.$ for the model chosen, where $\left.R_{E}=6371.2 \mathrm{~km}\right)$, while $R_{M S}$ is the radial distance of Saturn's subsolar magnetopause, which is estimated separately from each flyby data set (and $R_{S}=60330 \mathrm{~km}$ ). The $g_{1}^{0}$ factors are the axial dipole coefficients for Earth and Saturn, taken to be $g_{1 E}^{0}=-29682 \mathrm{nT}$, which is the 1995 International Geomagnetic Reference Field (IGRF) value (Barton, 1996), and $g_{1 S}^{0}=21160 \mathrm{nT}$, which is the SPV value. Thus, for example, for a typical subsolar magnetopause distance $R_{M S} \approx 20 R_{S}$ at Saturn, we find a field scaling factor $\boldsymbol{B}_{S}\left(\boldsymbol{r}_{S}\right) \sim-0.1 \boldsymbol{B}_{E}\left(\boldsymbol{r}_{E}\right)$. A typical field of $\sim 40 \mathrm{nT}$ in the near-Earth tail lobe thus transforms to a typical lobe field of $\sim 4 \mathrm{nT}$ at Saturn, which seems reasonable in terms of the flyby observations (e.g. Ness et al., 1981). 

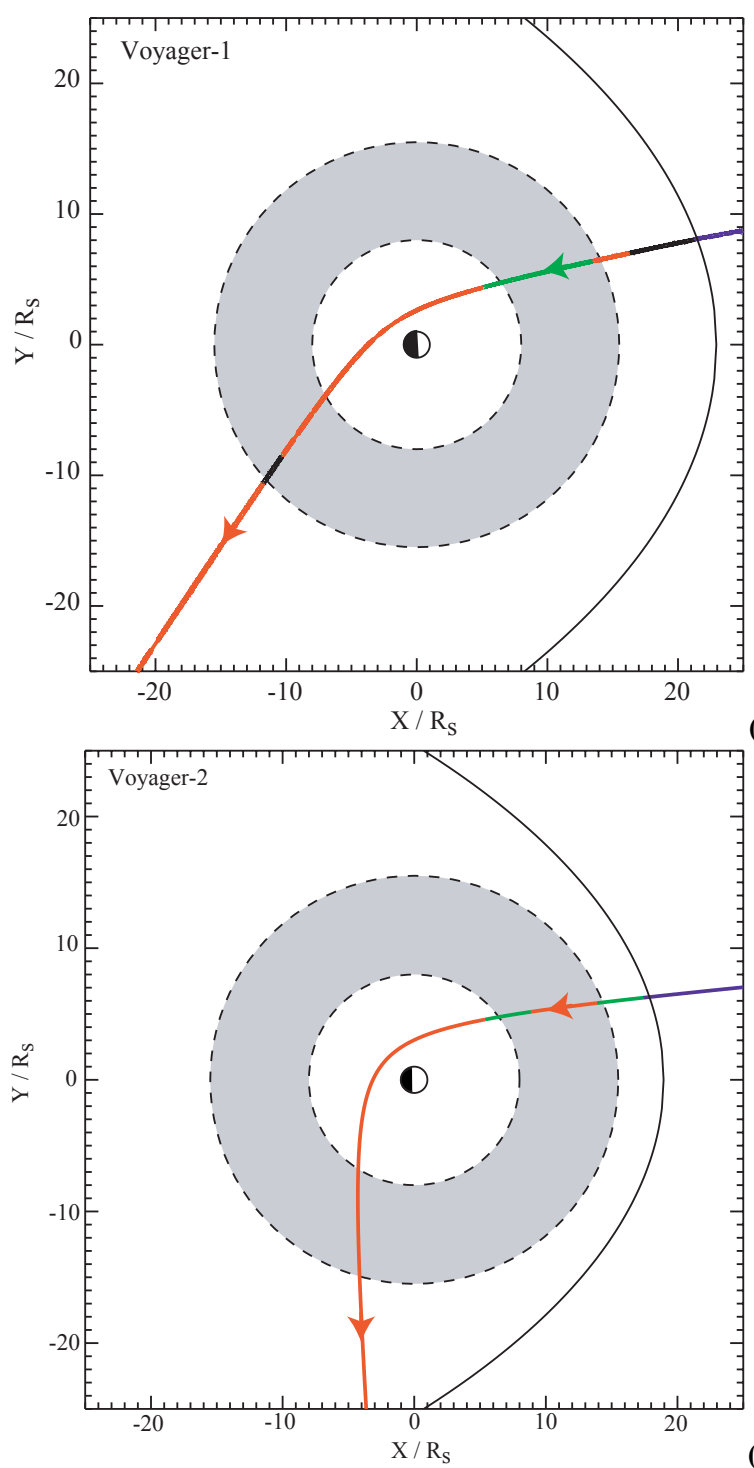

(a)

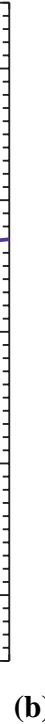

Fig. 3. Plots showing the colour-coded trajectories of (a) Voyager-1 and (b) Voyager-2, projected onto Saturn's equatorial plane, where $X$ points towards the Sun and $Y$ from dawn to dusk. The trajectories are colour-coded red when the observed azimuthal field is consistent with a 'lagging' field configuration (ignoring rapid fluctuations in the field), green when it is consistent with a 'leading' field configuration, and black when the azimuthal field is near-zero for an extended interval. The trajectories are colour-coded blue when the spacecraft is in the inbound magnetosheath. The black solid lines show model magnetopauses fitted to the observed magnetopause encounter(s) (the centre position of the five inbound magnetopauses in the case of Voyager-1), where we have employed the same magnetopause shape as used in the Tsyganenko field model. The stippled areas bounded by the dashed circles indicate the region of the Connerney et al. (1983) model ring current.

As indicated above, however, in the comparisons presented below we have tailored the magnetopause distances used in Eq. (6) to values consistent with the flyby data. We have also used a $D_{s t}$ value in the Earth model which, when transformed to Saturn using Eq. (6b), reproduces the observed near-planet strength of the axial perturbation field due to Saturn's ring current. However, we have not otherwise revised the terrestrial ring current model employed in the Tsyganenko model to reflect the ring current fields observed inside the kronian magnetosphere.

\section{Comparison of observed and calculated azimuthal fields}

\subsection{Spacecraft trajectories}

We begin our exposition of the Voyager data by first considering the flyby trajectories of the spacecraft, as they are germane to the results presented. Trajectory information is shown for Voyagers-1 and -2 in Figs. 3 and 4, respectively. Figure 3 shows colour-coded trajectories projected onto the equatorial plane, where $X$ points towards the Sun, and $Y$ from dawn to dusk. The black solid line shows a model magnetopause matched to the observed inbound magnetopause(s) (of the same shape as used in the Tsyganenko field model), and the stippled area bounded by the dashed lines indicates the ring current region in the Connerney et al. (1983) model. (The colour-coding on the trajectory refers to the nature of the azimuthal field observed, as will be discussed below, red for 'lagging', green for 'leading', black for near-zero, and blue for inbound magnetosheath.) It can be seen that both spacecraft entered the dayside magnetosphere in the immediate post-noon sector at $\sim 13: 00 \mathrm{LT}$, and then swung round the planet in the post-dusk sector, before exiting from the dawn magnetosphere, Voyager-1 at $\sim 03: 00$ LT and Voyager2 nearly along the dawn meridian. In terms of the mechanisms discussed above, therefore, we may expect to find either 'lagging' or 'leading' fields on the inbound passes, depending on the relative roles of the corotation-related and solar wind-related effects, though we note that the spacecraft were located quite close to the noon meridian where the solar wind effect goes to zero. However, only 'lagging' fields are expected on the outbound passes, resulting from some combination of both effects.

Figure 4 shows the trajectories (red-green-black-blue colour-coded) plotted in cylindrical co-ordinates on the $(\rho, z)$ plane, showing their relationship with the field and plasma structures. The black lines are magnetic field lines obtained by adding the field of the Connerney et al. (1983) ring current model to the SPV internal field. The model ring current flows within the area bounded by black dashed lines. It can be seen that Voyager-1 passed centrally through the model ring current region at relatively small $z$ values south of the equatorial plane on its inbound pass, reached closest approach at a distance $\sim 2 R_{S}$ south of the equator at a radial distance of 3.1 $R_{S}$, and then moved northward across the equator and through the ring current region again on its outbound pass. By contrast, Voyager-2 entered the magnetosphere with a significant displacement north of the equatorial plane, passed over the model ring current region on the 
inbound pass, crossed the equatorial plane near closest approach at a radial distance of $2.7 R_{S}$, and then exited with an even larger displacement south of the equatorial plane.

The blue solid lines in Fig. 4 then show plasma density contours, specifically of water-group ions (taken principally to be $\mathrm{O}^{+}$), derived from Voyager PLS data by Richardson and Sittler (1990) and Richardson (1995) (though we note that the analysis leading to these results used a dipole magnetic field only). Heavy-ion (rather than proton) contours are shown since this component contains the majority of the plasma mass on a given flux tube, and is hence the most dynamically significant. The innermost contour on the left of the diagram corresponds to an ion number density of $30 \mathrm{~cm}^{-3}$, with subsequent contours corresponding to $10,3,1,0.3$, and $0.1 \mathrm{~cm}^{-3}$. It is important to note that the heavy-ion plasma is confined to within $\sim 1 R_{S}$ of the equatorial plane at equatorial radial distances out to $\sim 8 R_{S}$. This results primarily from the large temperature anisotropy of the ions $\left(T_{\perp} \gg T_{\|}\right)$, together with low overall ion temperatures (few tens to $\sim 100 \mathrm{eV}$ ) and the confining centrifugal effect of the corotational flow. Since it is this plasma that will carry the majority of the equatorial radial current that closes the magnetosphere-ionosphere coupling current circuit in the inner part of the system, we may expect that when the spacecraft are located on field lines outside of, but threading through this plasma sheet, essentially the full azimuthal field given by Eq. (1) should be observed. This conclusion is reinforced by the finding by Richardson et al. (1998) that the principal heavy ion loss process in the region out to $\sim 8 R_{S}$ is $\mathrm{O}-\mathrm{O}^{+}$charge exchange, in which case we may expect the principal component of the radial current to be a 'pick-up' current associated with the newly-created ions. The north-south distribution of this current will then be determined by the distribution of the neutral species (derived from plasma sputtering of icy moons and micrometeorite erosion of ring grains), which is believed to be tightly confined within $\sim 0.5-1 R_{S}$ of the equatorial plane (e.g. Johnson et al., 1989; Pospieszalska and Johnson, 1991; Richardson et al., 1998). It can be seen from the figures that Voyager-1 was continuously located south of this plasma sheet for a considerable interval $(\sim 10 \mathrm{~h})$ around closest approach, before passing northward through the plasma sheet on its outbound pass, while Voyager-2 passed from the region north of the plasma sheet on its inbound pass, through the plasma sheet near closest approach, and then through the region south of the plasma sheet outbound. These represent prime intervals for the detection of the azimuthal fields of the magnetosphere-ionosphere coupling current system. Beyond $\sim 8 R_{S}$ the plasma structures shown in Fig. 4 may be representative of inbound dayside conditions only, since no plasma data are available at larger distances on the dawn outbound passes due to unfavourable spacecraft pointing geometries. However, the inbound data indicate that in this region the ions are more distributed along the field lines, due principally to reduced temperature anisotropies, together with increased temperatures and reduced angular velocities. Beyond $\sim 12 R_{S}$ the model densities are also highly spatially structured, reflecting the

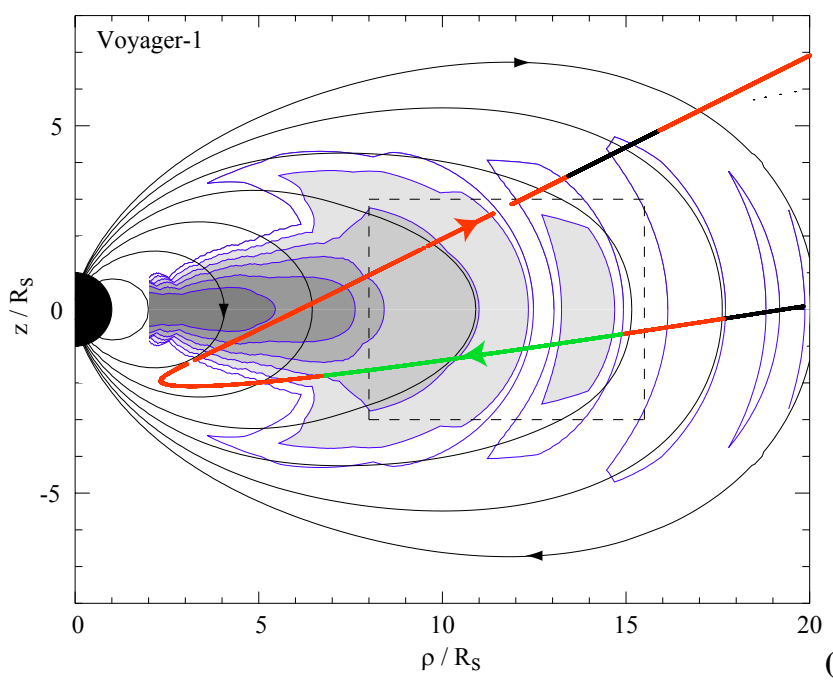

(a)

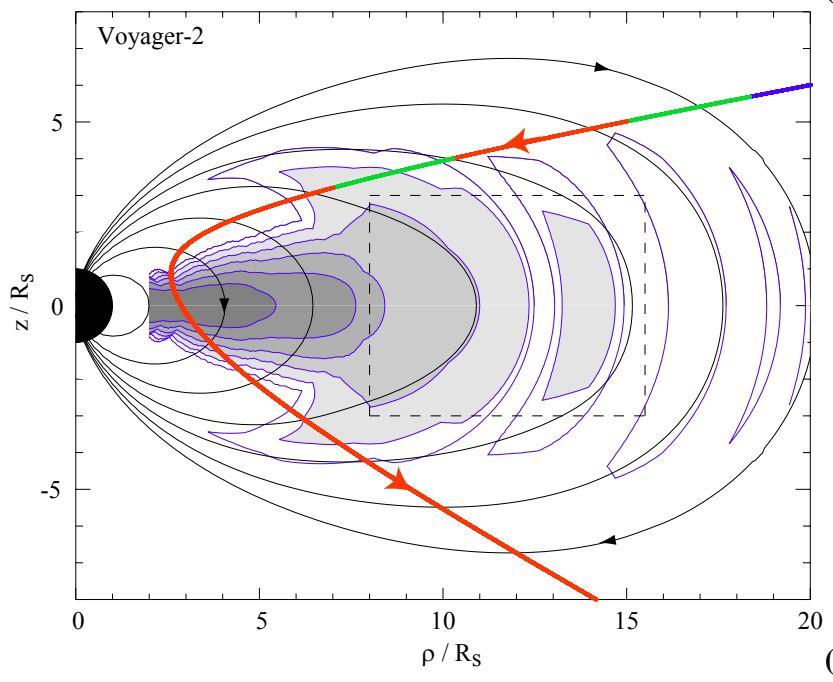

(b)

Fig. 4. Plots showing the colour-coded trajectories of (a) Voyager-1 and (b) Voyager-2, plotted in the $(\rho, z)$ plane. The colour coding is the same as in Fig. 3. The black solid lines show magnetic field lines determined from the SPV internal field model plus Connerney et al. (1983) ring current (black lines). The dashed lines show the cross section of the model ring-current region. The solid blue lines show contours of the heavy-ion $\left(\mathrm{O}^{+}\right)$density determined from the model of Richardson and Sittler (1990) and Richardson (1995), based on Voyager PLS plasma data. The innermost contour on the left of the diagram corresponds to an ion density of $30 \mathrm{~cm}^{-3}$ (with model values between 100 and $30 \mathrm{~cm}^{-3}$ being shown dark grey), with subsequent contours corresponding to densities of 10, 3, 1, 0.3, and $0.1 \mathrm{~cm}^{-3}$ (with values below $0.3 \mathrm{~cm}^{-3}$ being shown white).

structured plasma properties observed by the Voyagers in the outer dayside regions. According to the Richardson et al. (1998) plasma model, the principal $\mathrm{O}^{+}$'loss' process beyond $\sim 8 R_{S}$ is outward radial plasma diffusion, so that the principal component of the radial current will instead be that associated with outward transport, as at Jupiter outside of the Io torus. In this case the current may be more distributed with the plasma along the magnetospheric field lines, such 
that the azimuthal fields observed may then be reduced below that expected from Eq. (1), particularly on the Voyager-1 trajectory which is located closer to the equatorial plane than for Voyager-2.

\subsection{Comparison of observed and calculated azimuthal field for plasma sub-corotation}

We now turn to the data, and begin by comparing the observed azimuthal magnetic field with values calculated from Eq. (1) using the modelled plasma angular velocity profiles, as outlined in Sect. 2. Results for Voyager-1 and -2 are shown in Figs. 5 and 6, respectively, together with calculated values. The top three panels of each figure show the field components in cylindrical co-ordinates referenced to the planet's spin (and magnetic) axis, while the lower three panels show the corresponding spacecraft position also in cylindrical coordinates, specifically $\rho, z$, and local time. Each figure shows two (Earth) days of data, spanning the interval from the inbound magnetopause encounters near noon (leftmost vertical dashed line(s) marked 'MP'), through the closest approach to the planet (central vertical dashed line marked 'CA'), to radial distances of $\sim 20 R_{S}$ outbound in the dawn magnetosphere. The plots do not, therefore, encompass the outbound magnetopause crossings, which were multiple in both cases and occurred at (spherical) radial distances of $43-47 R_{S}$ for Voyager-1 and 50-70 $R_{S}$ for Voyager-2 (e.g. Behannon et al., 1983). For reference purposes, the top two panels in each figure display the radial and axial magnetic field components, respectively. The SPV internal model has been subtracted from these data, such that the fields shown are those due to external sources only. Following Connerney et al. (1983), some spurious values have been deleted near to the closest approach in each plot, where attitude reconstruction was insufficiently precise during spacecraft manoeuvers. The blue dotted curves in these panels show the field produced by the Connerney et al. (1983) ring current model, which can be seen to give a generally good account of these data. The same colour-code is also employed in the trajectory plots in Figs. 3 and 4, so that it is easy to judge the spatial relationships which exist between the behaviour of the azimuthal field and the poloidal field and plasma structures. The third panels in Figs. 5 and 6 then show the azimuthal field of central interest here, where we note that since the internal field is wholly poloidal, as indicated above, the data shown in this case represent the total azimuthal field measured. We further note that since the Connerney et al. (1983) ring current model is axisymmetric, it also produces no azimuthal field. The azimuthal field data are colour-coded red when, ignoring rapid fluctuations, the sense of the field is consistent with a 'lagging' configuration, green when it is consistent with a 'leading' configuration, and black when the mean field is close to zero for an extended interval. Blue indicates magnetosheath/magnetopause intervals. The dotted curves in this panel then show the azimuthal field computed for the plasma sub-corotation effect from Eq. (1), using the model parameters discussed in Sect. 2. As explained above, the value of the effective ionospheric Pedersen conductivity is treated essentially as a free parameter. The lower and upper blue dotted lines in these panels correspond to values of 1 and $2 \mathrm{mho}$, respectively.

Overall, it can be seen that the calculated fields for $\Sigma_{P}^{*}=$ $1-2$ mho encompass the observed values quite well when the latter are consistent with a 'lagging' configuration. However, it can also be seen that there are extended intervals, on the inbound passes particularly, where the observed field is instead consistent with a 'leading' configuration. In fact, careful examination of these plots in conjunction with Figs. 3 and 4 indicates a rather consistent picture for the two flybys. It can be seen first of all that there is a region extending inwards from the inbound magnetopause, where the azimuthal fields are either small and fluctuating, as for Voyager-1 near the equatorial plane, or are in the sense of a 'leading' field, as for Voyager- 2 well off the equatorial plane. These observations are then consistent in sense with field bending due to solar wind-related effects, an aspect that will be investigated quantitatively below. The azimuthal fields then reverse sense to become variable but typically 'lagging' and consistent with the calculated field for $\Sigma_{P}^{*} \approx 1-2$ mho in a region of the outer magnetosphere, where the plasma conditions are variable according to the plasma model of Richardson and Sittler (1990) and Richardson (1995). For Voyager-1 this region lies at cylindrical radial distances of $\sim 15-17 R_{S}$, while for Voyager-2 it is more extended radially but lies closer to the planet at $\sim 10-15 R_{S}$. Inside this region of relative agreement with Eq. (1) there then exists a further region of 'leading' fields, which is relatively weak for Voyager-1, located $\sim 1-2 R_{S}$ south of the equatorial plane, and relatively strong for Voyager-2, located $\sim 3-4 R_{S}$ north of the equatorial plane. We note from Figs. 3 and 4 that in both cases this corresponds to the interval when the spacecraft was passing through or immediately adjacent to the ring current region (as can also be seen from the variations in the $B_{z}$ field component in the upper panels of Figs. 5 and 6), where the plasma is more distributed along the field lines in the Richardson and Sittler (1990) and Richardson (1995) plasma model. We noted above that the 'lagging' fields due to the magnetosphere-ionosphere current could be weakened in this region, compared with that given by Eq. (1). The likelihood that the observed oppositely-directed 'leading' fields were produced by the solar wind effect will be investigated below. We note, however, that the azimuthal field variations observed during the inbound Voyager-2 pass were attributed to the 10-h periodic perturbation (of unknown origin) by Espinosa and Dougherty (2000).

It can also be seen in Figs. 5 and 6 that the observed and calculated azimuthal fields are also in reasonable agreement in the inner part of the magnetosphere. On the inbound pass this statement applies to the intervals when the spacecraft were located on field lines mapping to the equatorial plane within $\sim 8 R_{S}$ of the planet, and hence, were located on field lines threading the thin equatorial plasma sheet. The blue dotted curve in the fourth panel of the figures shows the equatorial crossing distance of the field line passing through the 

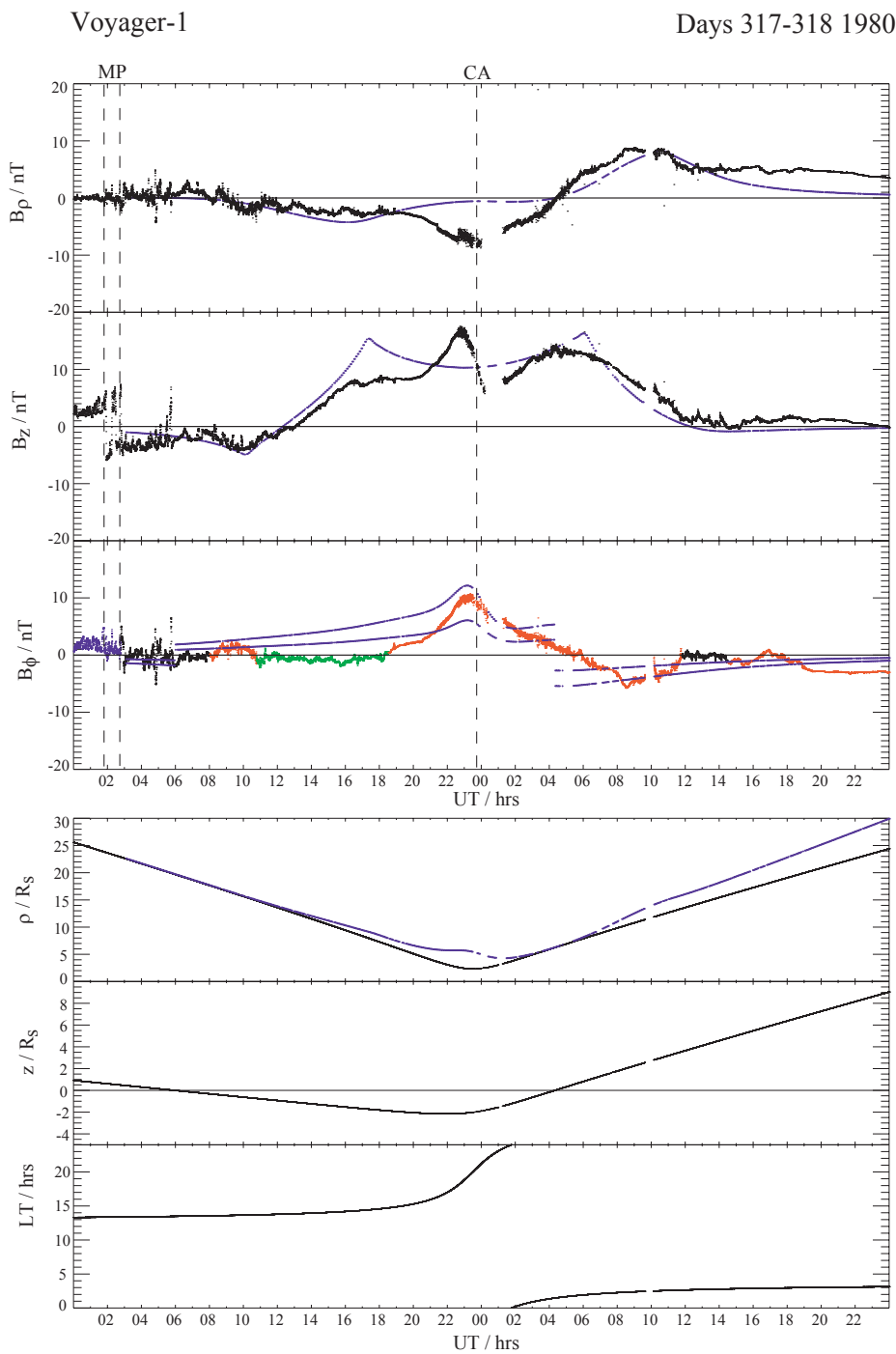

Fig. 5. Plot showing magnetic field and spacecraft position data for the Saturn flyby of Voyager-1. Two (Earth) days of data are shown, corresponding to days 317 and 318 of 1980 . The top three panels show the residual field components in cylindrical co-ordinates referenced to the planet's spin (and magnetic) axis, that is, from the top downwards, $B_{\rho}, B_{z}$, and $B_{\varphi}$ (in nT). These residuals are the measured field minus the SPV model of the internal planetary field (which is zero in the case of the azimuthal component). The azimuthal field data are colourcoded according to the implied sense of the field line bending. The data are colour-coded red when the field (ignoring rapid fluctuations) is consistent with a 'lagging' configuration, green when it is 'leading', and black when the field is on average close to zero. The data are colour-coded blue for magnetosheath/magnetopause intervals. The blue dotted lines in the top two panels show the components of the ring current field model derived by Connerney et al. (1983) (which again has zero azimuthal component). The model parameters are $R_{1}=8 R_{S}$, $R_{2}=15.5 R_{S}, D=3 R_{S}$, and $\mu_{o} I_{o} \approx 60.4 \mathrm{nT}$. The dotted lines in the panel showing the azimuthal magnetic field are calculated for the plasma sub-corotation effect using Eqs. (1)-(5), with $\Sigma_{P}^{*}$ equal to 1 mho (inner line) and 2 mho (outer line). The three lower panels give the position of the spacecraft in cylindrical co-ordinates, showing the cylindrical radial distance from the planet's spin (and magnetic) axis $\rho$, the distance along this axis from the equatorial plane $z$, and the local time (hours). The blue dotted line in the upper of these position panels shows the radial distance at which the field line passing through the spacecraft crosses the equatorial plane, determined from the SPV plus Connerney et al. (1983) ring current model employed here. The left-hand vertical dashed lines marked 'MP' show the positions of the first and last of five inbound magnetopause transitions, which occurred at spherical radial distances between 23 and $24 R_{S}$. The central vertical dashed line marked 'CA' shows the closest approach of the spacecraft to the planet, at a spherical radial distance of $3.1 R_{S}$.

spacecraft (according to the SPV plus Connerney et al. ring current model employed here). On the outbound pass reasonable agreement is found in the region of field lines mapping equatorially to $\sim 15 R_{S}$. The principal exceptions to these statements occur when the spacecraft were passing through the plasma sheet itself. Thus, for Voyager-1, a large 'lagging' field signature was observed throughout the closest approach, when the spacecraft was located south of the plasma sheet, peaking near the minimum cylindrical radial distance, with the magnitude of the azimuthal field then gradually declin- 

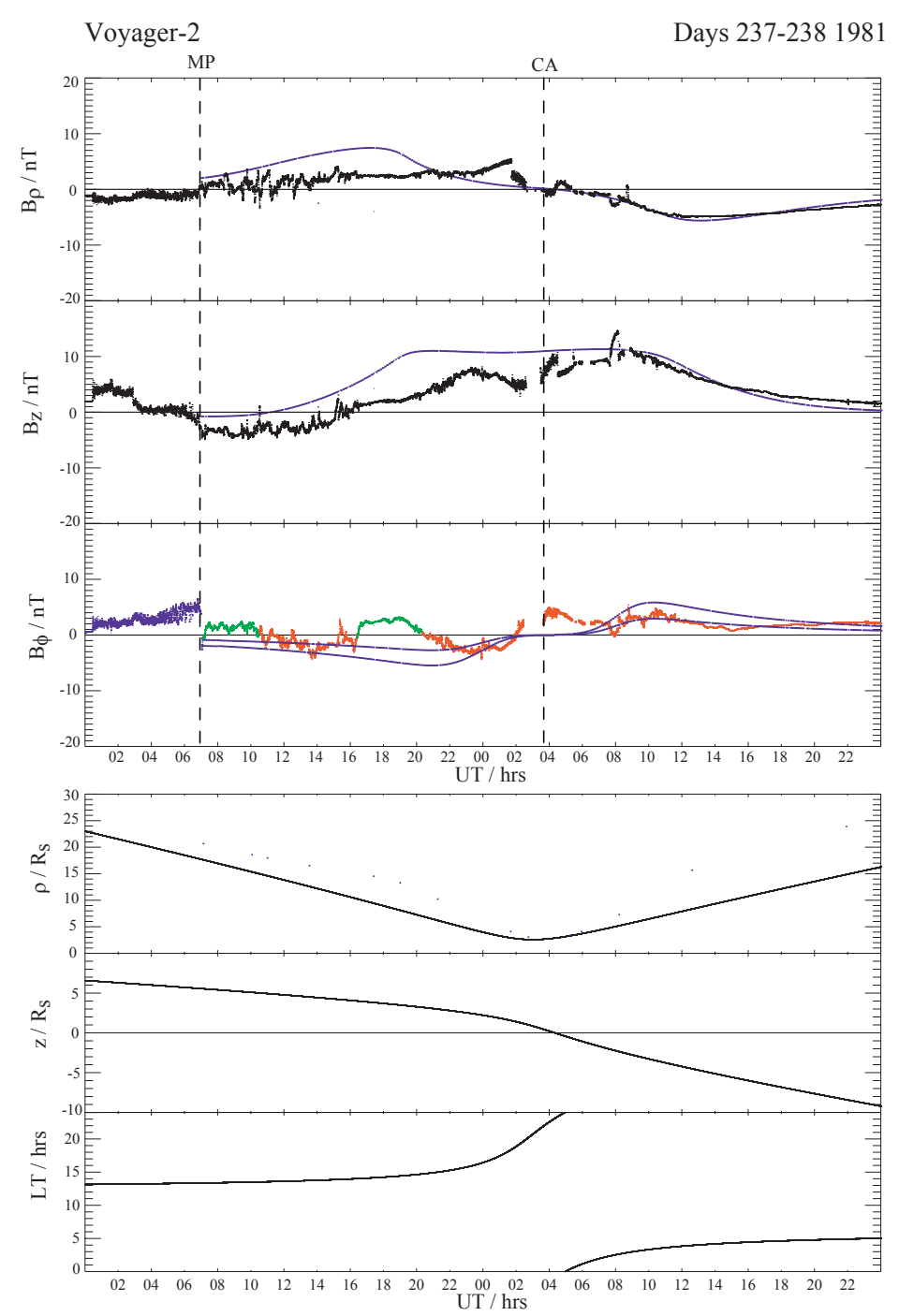

Fig. 6. As for Fig. 5, except for the Voyager-2 flyby. In this case the data correspond to days 237 and 238 of 1981 . The left-hand vertical dashed line shows the single inbound magnetopause crossing at $19 R_{S}$, while the central vertical dashed line shows closest approach at $2.7 R_{S}$.

ing and reversing in sense as the spacecraft moved through the plasma sheet on its outbound pass. By comparison, of course, the calculated field shows a sudden 'step' from one field sense to the other as the spacecraft crossed the equatorial plane. The observed field is significantly weaker than the calculated field in a region which is $\sim 1 R_{S}$ wide (northsouth), centred near (but northward of) the equatorial plane. In a related manner, the Voyager- 2 observations show the presence of 'lagging' fields essentially throughout the closest approach, with the azimuthal field reversing in sense as the spacecraft passed through the plasma sheet near the closest approach. However, the magnitude of the observed and calculated fields are smaller than for Voyager-1. In the calculation this results principally from the differing modelled angular velocity profiles employed, with the Voyager-2 profile remaining closer to near-rigid corotation to larger distances, as observed in the Voyager plasma data (see Fig. 4a of the companion paper) and as shown in Fig. 2. Closer agreement between observed and modelled fields could undoubtedly be achieved by detailed adjustment of the angular velocity models. However, such adjustment could not be justified in terms of $a$ priori fitting to the rather scattered angular velocity data, and has not been attempted here.

Beyond this region of reasonable agreement on the outbound passes, the observed azimuthal fields then fall in magnitude below those calculated to small (Voyager-2) or near-zero values (Voyager-1), before subsequently increasing again to larger magnitudes than those calculated, at (spacecraft) radial distances beyond $\sim 20 R_{S}$ for Voyager-1 and $\sim 15 R_{S}$ for Voyager-2. The extent of the contribution of the magnetopause and tail currents to these fields will now be investigated. 


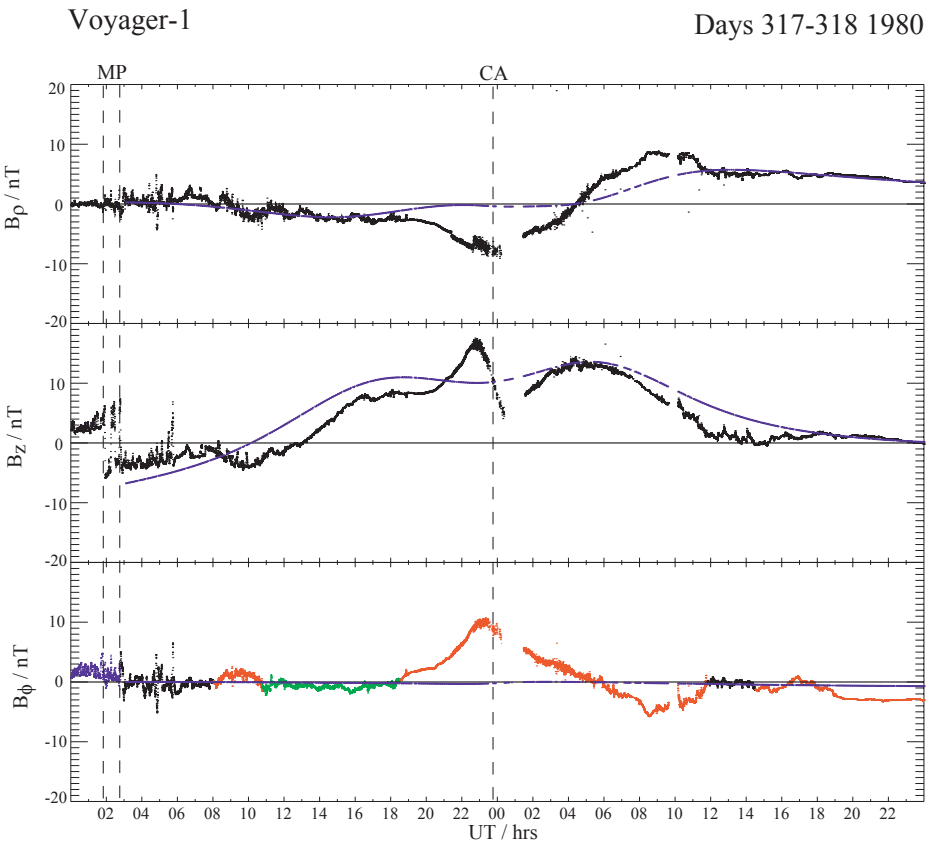

Fig. 7. Plot showing magnetic field data for the Saturn flyby of Voyager1 in cylindrical co-ordinates, as in the upper panels of Fig. 5, compared with a model field obtained by scaling the Tsyganenko $(1995,1996)$ model of the Earth's magnetic field as described in the text (blue dotted lines).

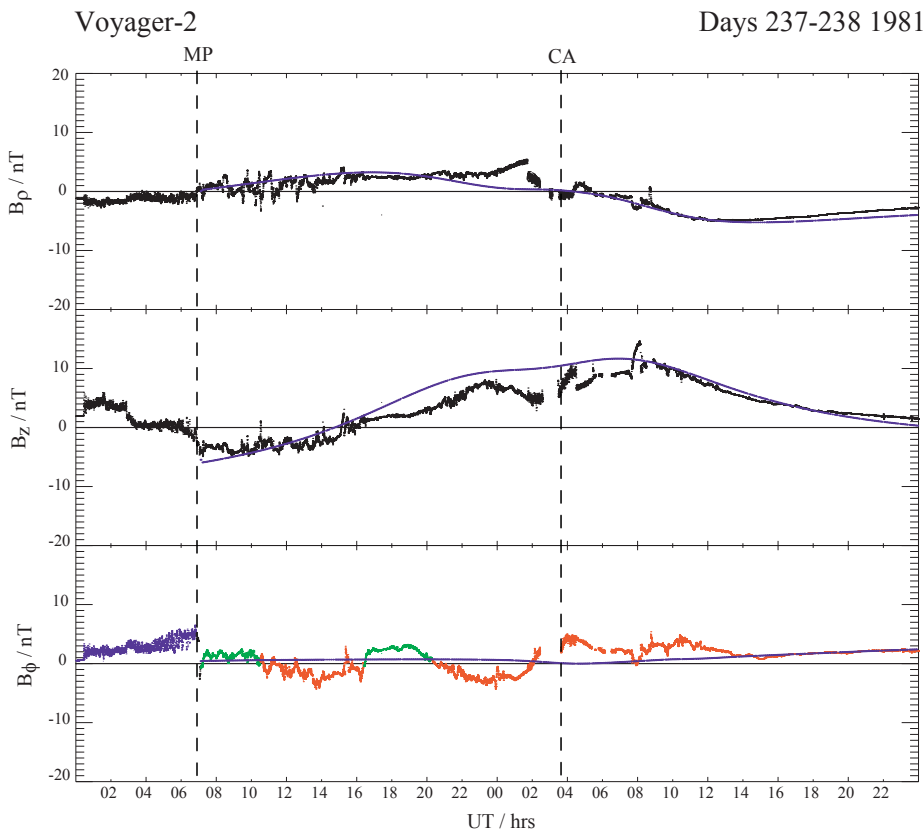

Fig. 8. As for Fig. 7, except for Voyager-2.

\subsection{Comparison of observed and calculated azimuthal} fields for the magnetopause-tail currents

We now estimate the contribution to the observed azimuthal field which is due to the magnetopause and tail current system, scaled from a model of the terrestrial magnetosphere, as outlined in Sect. 3. Results for Voyager-1 are shown in Fig. 7, in essentially the same format as the upper panels of Fig. 5, that is to say, the three panels of the plot show the residual poloidal components (with SPV internal model subtracted) and the colour-coded azimuthal component in cylin- drical co-ordinates, together with the scaled Earth field (blue dotted lines). In this case we have taken $R_{M S}=23.5 R_{S}$, roughly half way between the first and last observed nearnoon inbound magnetopause crossing. The position and field scaling given by Eq. (6) are then $\boldsymbol{r}_{E} \approx 0.47 \boldsymbol{r}_{S}$ (where the position vectors are in units of the corresponding planetary radii) and $\boldsymbol{B}_{S}\left(\boldsymbol{r}_{S}\right) \approx-0.075 \boldsymbol{B}_{E}\left(\boldsymbol{r}_{E}\right)$. We have also employed $D_{s t} \approx-130 \mathrm{nT}$ in the Earth model, which, with this field scaling, thus becomes a near-planet axial perturbation field of $+9.5 \mathrm{nT}$ at Saturn.

It can first be seen that, with the exception of the region 
in the immediate vicinity of the closest approach, the scaled Earth model reproduces the poloidal components at Saturn reasonably well. The implication of this result is that the spatial distribution of the ring current within the magnetosphere, relative to the magnetopause, is similar in the two cases. This is despite the fact that the physical mechanisms leading to the existence of the ring currents are believed to be different in the two systems, involving solar wind-driven convection in the Earth's case, and rotation and radial diffusion at Saturn. As a by-product of this investigation, therefore, we note that a future approach to three-dimensional field modelling at Saturn via modification of Earth models appears feasible. The important point for this study, however, is the implication that the structure of the modelled field captures the essential features of the poloidal field at Saturn. However, this is by no means true of the azimuthal components shown in the lower panel. During the inbound pass, for example, the value of the modelled azimuthal field is negative, implying a 'leading' field configuration in the post-noon sector as expected for the solar wind-related effect (Fig. 1). However, because the spacecraft was located close to the noon meridian, as pointed out above, and also relatively close to the equatorial plane, the magnitude of the modelled field is very small, remaining less than $\sim 0.5 \mathrm{nT}$ throughout the inbound interval. The modelled field thus does not explain even the rather weak 'leading' fields observed in the ring current region on the inbound pass, with the implication that these are probably due to an effect other than field bending associated with the 'fringing' field of the magnetopause-tail current system. Similarly, the results for the outbound pass indicate that the latter current system makes essentially no contribution to the 'lagging' fields observed out to distances of $\sim 15 R_{S}$. Beyond this distance, the modelled field increases to become an increasing fraction of the observed field as the spacecraft nears the 'tail' region (see also the good agreement with the radial field component), until finally reaching agreement with the observed field in the region adjacent to the outbound magnetopause (data not shown). We note that the position of the outbound magnetopause agrees with the model values to within $10 \%$.

Results for Voyager-2 are shown in the same format in Fig. 8. In this case we have taken $R_{M S}=19 R_{S}$, in agreement with the position of the inbound magnetopause crossing. We note that a major magnetospheric expansion took place at some point during the flyby due to immersion in the jovian magnetic tail (Behannon et al., 1983), but the time at which this occurred is unknown, and modelling the expanded state (with weaker azimuthal fields) has not been attempted here. With this value of $R_{M S}$, then, the position and field scaling given by Eq. (6) are $\boldsymbol{r}_{E} \approx 0.58 \boldsymbol{r}_{S}$ and $\boldsymbol{B}_{S}\left(\boldsymbol{r}_{S}\right) \approx-0.14 \boldsymbol{B}_{E}\left(\boldsymbol{r}_{E}\right)$, respectively. We have also employed $D_{s t} \approx-50 \mathrm{nT}$ in the Earth model, which thus becomes a near-planet axial perturbation field of $+7 \mathrm{nT}$ at Saturn. Again, it can be seen that the scaled Earth model reproduces the poloidal components at Saturn quite well. However, while the modelled azimuthal components are larger than before, they are still not large enough to explain the 'leading' fields observed during the inbound pass, either in the region next to the magnetopause, or in the region adjacent to the ring current. The additional presence of other dynamical effects is thus again indicated, certainly in the latter case. On the outbound pass, the modelled fields are again too small to make a significant contribution out to radial distances of $\sim 10 R_{S}$, but then become comparable to the observed azimuthal field at distances beyond.

Overall, these results suggest that the azimuthal magnetic fields which are due to the magnetopause-tail current system are not a significant effect during the dayside inbound passes, though they would need to be included in any careful treatment, as indicated by the Voyager- 2 results. They also do not contribute significantly within radial distances of $\sim 10$ $15 R_{S}$ on the dawn-side outbound passes, where the observed azimuthal fields are generally the largest. However, beyond such distances they gradually become the largest azimuthal component as the spacecraft approaches and enters the region dominated by the fields of the magnetospheric tail.

\section{Summary and discussion}

In this paper we have for the first time quantitatively examined two mechanisms that will produce azimuthal magnetic fields in Saturn's magnetosphere, and have compared the calculated fields with those observed during the Saturn flybys of the two Voyager spacecraft. The first mechanism is associated with the magnetosphere-ionosphere coupling currents that result from sub-corotation of the magnetospheric plasma, which produce 'lagging' fields at all local times associated with the transfer of angular momentum from the atmosphere to the plasma. The second is associated with the asymmetric confinement of the planetary field by the solar wind, i.e. the effect of the magnetopause and tail current system, which causes the field lines to be bent away from the noon meridian on either side. Our calculation of the expected azimuthal field which is due to plasma sub-corotation is based on plasma angular velocity profiles observed during the Voyager flybys, combined with a model of the magnetic field, also derived from Voyager data, which is used to map the angular velocities into the ionosphere. The effects of the magnetopause and tail current system have been estimated empirically by scaling a data-based terrestrial field model to Saturn.

A principal conclusion of the study is that the azimuthal fields observed in the inner part of the magnetosphere on both flybys can be reasonably well accounted for by the magnetosphere-ionosphere coupling mechanism, given an effective value of the ionospheric Pedersen conductivity of $\sim 1-2$ mho. The magnetopause-tail current system contributes only small fields within this region. Our conclusion applies specifically to the region of field lines mapping equatorially to distances within $\sim 8 R_{S}$ of the planet on the dayside inbound passes, and to distances within $\sim 15 R_{S}$ on the dawn side outbound passes. Voyager data indicate that the plasma distribution within $\sim 8 R_{S}$ consists principally of a 
thin equatorial heavy-ion plasma sheet lying within $\sim 1 R_{S}$ of the equatorial plane, as shown in Fig. 4. We thus infer that the main intervals during which reasonable agreement has been found correspond to intervals when the spacecraft were magnetically connected to, but lying outside of, this plasma sheet where the radial closure current flows, such that they were then observing essentially the full azimuthal field of the magnetosphere-ionosphere coupling currents. Our results suggest that such conditions may extend to $\sim 15 R_{S}$ on the dawn side, beyond the region where dawn plasma data were obtained. With the sub-corotation interpretation of the observed azimuthal fields in the inner region, therefore, we thus infer an effective value of the Pedersen conductivity in the conjugate ionosphere of $\sim 1-2$ mho.

In drawing this conclusion we should recognise the possibility, however, that other magnetosphere-ionosphere coupling currents could also contribute to the fields observed in the inner region. For example, Connerney et al. (1983) modelled the principal azimuthal magnetic field signature observed near the closest approach by Voyager-1 in terms of a line current flowing into the nightside auroral zone at $80^{\circ}$ southern latitude and 21:30 LT, and discussed its presence in terms of solar wind-magnetosphere coupling effects. This model of the current certainly has the virtue that it also provides some explanation of the additional field perturbations that can be seen near closest approach in the poloidal components in Fig. 5, which are not accounted for in the axisymmetric model derived here. The important point we wish to make, however, is that to the extent that the observed fields are actually due to other causes, the contribution which is due to sub-corotation of the plasma must be less, thus implying a smaller value of the effective ionospheric Pedersen conductivity. Given the degree to which our modelled fields account in a simple way for the major features of the 'closest approach' data on both flybys, it seems likely to us that the sub-corotation coupling currents play a significant role in this region, with a consequent inferred effective Pedersen conductivity of the order of $\sim 1$ mho. If we take the opposite view, however, that the observed azimuthal fields in this region are principally due to other physical causes, for example, along the lines discussed by Connerney et al. (1983), then we must instead infer that the effective value of the Pedersen conductivity must be significantly less than such values, such that $\sim 1-2$ mho represents a robust upper limit. In either case, the results of this study show that values above $\sim 2$ mho, i.e. in the range $10-100$ mho, can definitely be ruled out.

Outside of the inner region, the plasma in the dayside magnetosphere is believed to be more distributed along the field lines according to present models, such that the inbound spacecraft were then presumably immersed within the current-carrying magnetospheric plasma. Intervals of both 'leading' and 'lagging' azimuthal fields were observed in this region during the inbound passes of both spacecraft. 'Leading' (or near-zero) fields were observed in a layer $\sim 5 R_{S}$ wide adjacent to the magnetopause, and within (or adjacent to) the ring current region at equatorial radial distances between $\sim 8$ and $\sim 15 R_{S}$, with a region of 'lagging' fields lying between. The magnitude of the lagging fields was again found to be consistent with those calculated for sub-corotation of the plasma, with an effective value of the ionospheric Pedersen conductivity of $\sim 1-2$ mho, though given the intermittent nature of these fields it seems unclear whether or not this agreement is fortuitous. The magnitude of the observed 'leading' fields is found to be significantly larger than those calculated from the scaled terrestrial model, indicating that additional dynamical processes are at work, perhaps related to the spin-periodic effect discussed by Espinosa and Dougherty (2000). On the dawn side outbound passes, the observed azimuthal field fell below values calculated for the sub-corotation mechanism on field lines mapping equatorially beyond $\sim 15 R_{S}$, before subsequently increasing once more to 'lagging' values greater than those calculated at radial distances beyond $\sim 20 R_{S}$. Our results indicate that the latter fields are simply those due to the tail current system, as might reasonably be expected.

Overall, our study has raised a number of issues concerning the origins of azimuthal magnetic fields inside Saturn's magnetosphere. Specifically, we have shown that azimuthal fields associated with sub-corotation of the plasma and angular momentum transfer from the atmosphere to the magnetosphere may play a substantial role, particularly in the inner regions located outside of, but mapping into, the thin equatorial heavy-ion plasma sheet. Our results indicate that the magnetopause-tail current system leads to little field bending in this inner region. If we then assume that the majority of the azimuthal field which is measured in this region is due to plasma sub-corotation, the implied value of the effective Pedersen conductivity of Saturn's ionosphere is $\sim 1-$ 2 mho. If, on the other hand, we assume that the azimuthal field has substantial contributions from other sources, then the latter values provide an upper limit. Outside of this inner region, both 'lagging' and 'leading' fields were observed on the dayside, but the 'leading' fields are generally too large to be accounted for by the fringing fields of the magnetopausetail current system, and may instead be principally related to the dynamical process producing the $\sim 11 \mathrm{~h}$ periodicity in the field data found by Espinosa and Dougherty (2000). We also note the presence of small-scale features in the field data which may be associated with ultra low frequency (ULF) waves. In the dawn and nightside magnetosphere, the field of the tail current system becomes significant at radial distances beyond $\sim 15-20 R_{S}$. We finally note, as a by-product, that the results of this study also indicate that modification and scaling of terrestrial field models may form a useful future approach to three-dimensional magnetic field modelling at Saturn.

Acknowledgements. We would like to thank Joe Mafi at the Planetary Data Center, UCLA, for supplying us with plasma and magnetic field data from the Voyager Saturn flybys. We would also like to thank Dr J. D. Richardson for supplying the code which generated the plasma density contour plots shown in Fig. 4. EJB and JAW were supported during the course of this study by PPARC Grant PPA/G/O/1999/00181, while SWHC was supported by PPARC Se- 
nior Fellowship PPA/N/S/2000/00197.

Topical Editor T. Pulkinnen thanks two referees for their help in evaluating this paper.

\section{References}

Acuña, M. H., Behannon, K. W., and Connerney, J. E. P.: Jupiter's magnetic field and magnetosphere, in Physics of the Jovian Magnetosphere, edited by Dessler, A. J., p. 1, Cambridge Univ. Press, Cambridge, UK, 1983.

Atreya, S. K., Waite, Jr., J. H., Donahue, T. M., Nagy, A. S., and McConnell, J. C.: Theory, measurements and model of the upper atmosphere and ionosphere of Saturn, edited by Gehrels, T. and Matthews, M. S., p. 239, Univ. Arizona Press, Tucson, Arizona, USA, 1984.

Barton, C. E.: Revision of the International Geomagnetic Reference Field, EOS Transaction, 77, 16, 1996.

Behannon, K. W., Lepping, R. P., and Ness, N. F.: Structure and dynamics of Saturn's outer magnetosphere and boundary regions, J. Geophys. Res., 88, 8791, 1983.

Bunce, E. J. and Cowley S. W. H.: Divergence of the equatorial current in the dawn sector of Jupiter's magnetosphere: analysis of Pioneer and Voyager magnetic field data, Planet. Space Sci., 49, 1089, 2001.

Cheng, A. F. and Waite, Jr., J. H.: Corotation lag of Saturn's magnetosphere: Global ionospheric conductivities revisited, J. Geophys. Res., 93, 4107, 1988.

Connerney, J. E. P., Ness, N. F., and Acuña, M. H.: Zonal harmonic model of Saturn's magnetic field from Voyager 1 and 2 observations, Nature, 298, 44, 1982.

Connerney, J. E. P., Acuña, M. H., and Ness, N. F.: Currents in Saturn's magnetosphere, J. Geophys. Res., 88, 8779, 1983.

Connerney, J. E. P., Acuña, M. H., and Ness, N. F.: The $Z_{3}$ model of Saturn's magnetic field and the Pioneer 11 vector helium magnetometer observations, J. Geophys. Res., 89, 7541, 1984.

Cowley, S. W. H. and Bunce E. J.: Origin of the main auroral oval in Jupiter's coupled magnetosphere-ionosphere system, Planet. Space Sci., 49, 1067, 2001.

Cowley, S. W. H. and Bunce E. J.: Corotation-driven magnetosphere-ionosphere coupling currents in Saturn's magnetosphere and their relation to the auroras, Ann. Geophysicae, in press, 2003.

Davis, Jr., L. and Smith, E. J.: A model of Saturn's magnetic field based on all available data, J. Geophys. Res., 95, 15 257, 1990.

Espinosa, S. A. and Dougherty, M. K.: Periodic perturbations in Saturn's magnetic field, Geophys. Res. Lett., 27, 2785, 2000.

Fairfield, D. H.: Average magnetic field configuration of the outer magnetosphere, J. Geophys. Res., 73, 7329, 1968.

Frank, L. A., Burek, B. G., Ackerson, K. L., Wolfe, J. H., and Mihalov, J. D.: Plasmas in Saturn's magnetosphere, J. Geophys. Res., 85, 5695, 1980.

Hill, T. W.: Inertial limit on corotation, J. Geophys. Res., 84, 6554, 1979.

Hill, T. W.: The jovian auroral oval, J. Geophys. Res., 106, 8101,
2001.

Huang, T. S. and Hill, T. W.: Corotation lag of the jovian atmosphere, ionosphere and magnetosphere, J. Geophys. Res., 94, 3761, 1989.

Johnson, R. E., Pospieszalska, M. K., Sittler, Jr., E. C., Cheng, A. F., Lanzerotti, L. J., and Sieveka, E. M.: The neutral cloud and heavy ion inner torus at Saturn, Icarus, 77, 311, 1989.

Khurana, K. K.: Influence of solar wind on Jupiter's magnetosphere deduced from currents in the equatorial plane, J. Geophys. Res., 106, 25 999, 2001.

Khurana, K. K. and Kivelson, M. G.: Inference of the angular velocity of plasma in the jovian magnetosphere from the sweepback of magnetic field, J. Geophys. Res., 98, 67, 1993.

Maurice, S. and Engel, I. M.: Idealized Saturn magnetosphere shape and field, J. Geophys. Res., 100, 17 143, 1995.

Ness, N. F., Acuña, M. H., Lepping, R. P., Connerney, J. E. P., Behannon, K. W., Burlaga, L. F., and Neubauer, F.: Magnetic field studies by Voyager 1: Preliminary results at Saturn, Science, 212, 211, 1981.

Pospieszalska, M. K. and Johnson, R. E.: Micrometeoritic erosion of main rings as a source of plasma in the inner Saturnian plasma torus, Icarus, 93, 45, 1991.

Richardson, J. D.: Thermal ions at Saturn: plasma parameters and implications, J. Geophys. Res., 91, 1381, 1986.

Richardson, J. D.: A new model for plasma transport and chemistry at Saturn, J. Geophys. Res., 97, 13 705, 1992.

Richardson, J. D.: An extended plasma model for Saturn, Geophys. Res. Lett., 22, 1177, 1995.

Richardson, J. D. and Sittler, Jr., E. C.: A plasma density model for Saturn based on Voyager observations, J. Geophys. Res., 95, 12 019, 1990.

Richardson, J. D., Eviatar, A., McGrath, M. A., and Vasyliunas, V. M.: OH in Saturn's magnetosphere: Observations and theory, J. Geophys. Res., 103, 20 245, 1998.

Smith, E. J., Davis, Jr., L., and Jones, D. E.: Jupiter's magnetic field and magnetosphere, in Jupiter, edited by Gehrels, T., p. 788, University of Arizona Press, Tucson, USA, 1976.

Trauger, J. T., Clarke, J. T., Ballester, G. E., Evans, R. W., Burrows, C. J., Crisp, D., Gallagher, J. S., III, Griffiths, R. E., Hester, J. J., Hoessel, J. G., Holtzman, J. A., Krist, J. E., Mould, J. R., Sahai, R., Scowen, P. A., Stapelfeldt, K. R., and Watson, A. M.: Saturn's hydrogen aurora: Wide field and planetary camera 2 imaging from the Hubble Space Telescope, J. Geophys. Res., 103, $20237,1998$.

Tsyganenko, N. A.: Modeling the Earth's magnetospheric magnetic field within a realistic magnetopause, J. Geophys. Res., 100, 5599, 1995.

Tsyganenko, N. A.: Effects of the solar wind conditions on the global magnetospheric configuration as deduced from data-based field models, in: Third International Conference on Substorms (ICS-3), ESA SP-389, p. 181, ESA Publ., Noordwijk, The Netherlands, 1996.

Vasyliunas, V. M.: Plasma distribution and flow, in: Physics of the Jovian Magnetosphere, edited by Dessler, A. J., p. 395, Cambridge Univ. Press, Cambridge, UK, 1983. 